\title{
Serum Choice Influences Lipid Accumulation and Cell Viability in Fatty Acid Treated Immortalised Hepatocytes and Hepatic Stellate Cells
}

\author{
Z. Zhang, J.L. Thorne and J.B. Moore \\ School of Food Science and Nutrition, University of Leeds, Leeds, LS2 9JT
}

Non-alcoholic fatty liver disease (NAFLD) is now the most common cause of liver disease in developed countries ${ }^{(1)}$. Previous research has shown that vitamin D may reduce the inflammatory and pro-fibrogenic activity of hepatic stellate cells (HSCs) in vitro ${ }^{(2)}$. However, the mechanisms underpinning the role of vitamin D in NAFLD pathogenesis, especially in HSCs, are not fully understood. With an overall objective of establishing a lipid loading model in immortalised hepatocytes (HepG2 cells) and HSCs (LX-2 cells); the aim of these experiments was to test the cell's responses to different fatty acid doses when cultured in either serum-containing medium (SCM), serum-free medium (SFM) or charcoal-stripped serum-containing medium (CSM).

Cells were routinely cultured in SCM and seeded to reach approximately $80 \%$ confluence prior to experimental treatment. Then, to test if there was interference from lipophilic materials contained in the serum, the SCM was either replenished, replaced with CSM, or replaced with SFM, and cells cultured for $16 \mathrm{hrs}$ in the fresh media prior to $6 \mathrm{~h}$ or $24 \mathrm{~h}$ fatty acid treatment $(0-500 \mu \mathrm{M}, 1: 1 \mathrm{oleic}$ acid: palmitic acid). Nile red was used to measure total intracellular lipid, while cell viability was measured by MTT assay. Data were quantified relative to the cells cultured in SCM at each concentration and analysed by two-way ANOVA with Holm-Sidak test.

A significant effect of medium choice on cell viability was observed, with reductions seen in both cell lines treated in SFM at both timepoints (HepG2 $\mathrm{n}=3,6 \mathrm{~h} \mathrm{P}=0.0304,24 \mathrm{~h} \mathrm{P}<0.0001$; LX-2 $\mathrm{n}=5,6 \mathrm{~h} \mathrm{P}<0.0001,24 \mathrm{~h} \mathrm{P}<0.0001$ ). Loss of viability was particularly pronounced in LX-2 cells cultured in SFM for $24 \mathrm{~h}$, where an $\sim 40 \%$ reduction was observed $(\mathrm{P}<0.0001)$ independent of any effects of fatty acid dose on viability $(\mathrm{P}=0.3533)$. On the other hand, no differences in viability were detected between cells cultured in CSM compared to SCM.Fatty acid treatment led to dose-dependent intracellular lipid accumulation in both cell lines, however, effects from serum were observed. After $6 \mathrm{~h}$ of fatty acid treatment, cells treated in SFM or CSM had accumulated less lipid relative to SCM cultured cells $(\mathrm{HepG} 2 \mathrm{n}=3, \mathrm{LX}-2 \mathrm{n}=5$, both $\mathrm{P}<0.0001)$. While this reduction remained at $24 \mathrm{~h}$ for cells treated in SFM, there was no difference in lipid loading observed between SCM and CSM at this time point (HepG2 n = 3 P = 0.0379; LX-2 n = 5 P $<$ $0.0001)$.

In summary, these experiments show CSM is a better choice, resulting in optimal cell viability and higher lipid loading in both cell lines after $24 \mathrm{~h}$ fatty acid treatment. Our results underscore the importance of serum choice in for in vitro lipid loading experiments. Using CSM for vitamin D and fatty acid cotreatment, ongoing experiments are examining mRNA, protein, and microRNA expression in hepatocytes and HSCs in response to vitamin D and lipid loading cotreatment.

1. Moore JB (2019) Proc Nutr Soc 78, 290-304.

2. Udomsinprasert W \& Jittikoon J (2019) Biomed Pharmacother 109, 1351-60. 